This is a self-archived version of an original article. This version may differ from the original in pagination and typographic details.

Author(s): Rinta-Antila, Sami; Trzaska, Wladyslaw; Rissanen, Juho

Title: Conversion electron spectroscopy at IGISOL

Year: 2014

Version: Accepted version (Final draft)

Copyright: (c) Springer Science+Business Media B.V. 2012.

Rights: In Copyright

Rights url: http://rightsstatements.org/page//nC/1.0/?language=en

Please cite the original version:

Rinta-Antila, S., Trzaska, W., \& Rissanen, J. (2014). Conversion electron spectroscopy at IGISOL. Hyperfine Interactions, 223(1-3), 73-80. https://doi.org/10.1007/s10751-012-0606-7 


\title{
Conversion electron spectroscopy at IGISOL
}

\author{
Sami Rinta-Antila - Wladyslaw Trzaska • \\ Juho Rissanen
}

Received: date / Accepted: date

\begin{abstract}
Conversion elecron spectroscopy has been an important part of the nuclear spectrocopy research at the Department of Physics of the University of Jyväskylä since the commissioning of the first cyclotron in the mid 1970s. At the IGISOL facility a specialiced conversion electron spectrometer ELLI was developed in the late 1980s. The first results with ELLI were obtained using the beams from the old MC-20 cyclotron to study newly discovered isotopes of refractory fission products. In the present K130 cyclotron laboratory ELLI has been utilized in many decay-spectroscopy experiments both neutron-deficient and neutron-rich side of the valley of stability. In the early 2000 s the new JYFLTRAP ion trap system overthrew ELLI from its permanent place in the IGISOL beamline. Conversion electron spectroscopy has continued with the new Penning trap that has been used in in-trap electron spectroscopy tests and post-trap electron spectroscopy is foreseen.
\end{abstract}

Keywords Conversion electron spectroscopy - On-line mass separator · Trap-assisted spectroscopy

\section{Introduction}

Nuclear spectroscopy was the dominant research line at the Department of Physics, University of Jyväskylä (JYFL) already in the early days of the accelerator physics there. In that respect JYFL was basically following the world-wide boom made possible by the availability of commercial germanium detectors. However, what set JYFL apart, already at that time, was the equal research effort devoted to the electron spectroscopy. For instance, during the first full year of operation of the MC-20 cyclotron (1976) 60 of the total of 167 days of beam on target (36\%) were used for in-beam gamma-ray spectroscopy

S. Rinta-Antila

Department of Physics, 40014 University of Jyväskylä, Finland

E-mail: sami.rinta-antila@phys.jyu.fi 
and 55 days $(33 \%)$ for in-beam electron spectroscopy. Similar ratio between gamma and electron measurements prevailed throughout the late 1980s. Naturally, it has been self evident that low-energy transitions are heavily converted and that in numerous cases measuring the conversion electron spectra is not just the best way but the only way of looking for exotic excited states or finding the missing transitions. But, to our knowledge, no other laboratory had such a pronounced commitment to the electron spectroscopy as JYFL in the late 1970s and 1980s.

\section{Electron Spectroscopy at JYFL}

Unlike in the case of gamma-ray detection, there are no off-the-shelf solutions for high-resolution electron measurements. The first precision electron spectrometers were developed in Sweden in the mid 1950s. This pioneering role was recognized by the Nobel Committee by awarding in 1981 the Prize in Physics to Kai M. Siegbahn, together with Nicolaas Bloembergen and Arthur L. Schawlow. Indeed, what gave JYFL a considerable head start in electron spectroscopy were the magnetic spectrometers obtained from Sweden [1] and the passion of the late Prof. Juhani Kantele, one of the founders of JYFL and the first chair in experimental nuclear physics in Finland. The main methodological breakthrough that made conversion electron spectroscopy a viable addition to gamma-ray measurements was made in parallel at the tandem laboratory in Uppsala [2] and at JYFL [3]. It was accomplished by combining a magnetic spectrometer operating in a broad-band lens mode with a modern semiconductor detector. The lens mode secured very good background suppression, the broad transmission provided high efficiency, and the excellent resolution was guaranteed by the cooled detector. The most successful of these combination spectrometers was a Siegbahn-Slätnis type intermediate-image magnetic lens beta-ray spectrometer that came to JYFL via Åbo Akademi around 1980. After minor modifications and with a new power supply and a $5 \mathrm{~mm}$ thick $\mathrm{Ge}(\mathrm{HP})$ in the focal plane this device has established the highenergy record in conversion electron spectroscopy by measuring E0 transitions in ${ }^{208} \mathrm{~Pb}$ at above $5 \mathrm{MeV}$ of excitation energy $[4,5]$.

\section{First conversion electron measurements at IGISOL}

The invention of IGISOL [6] in 1982 has opened new research opportunities at JYFL. In particular, the possibility to mass separate even the short-lived fission products enabled the production of radioactive ion beams in a very broad mass range. What followed very quickly was the discovery of many new, neutron-rich isotopes, especially in the region $42 \leq Z \leq 47$. The identification of those isotopes was made by well established spectroscopic techniques. At first the use of a plain silicon detector was sufficient to obtain adequate conversion electron spectra. Mass separated sources, the use of tape transport 
to remove buildup of activities and coincidence requirements yielded adequate background reduction during the first experiments. Already this simple approach resulted in many new discoveries in the mass region $110 \leq A \leq 117$ [7]. Nevertheless, by the end of 1980 it became obvious that IGISOL needed a dedicated electron spectrometer.

By that time a significant progress was achieved in electron spectroscopy. In addition to the bulky Siegbahn-type spectrometers with massive iron yokes and powerful current supplies several new technologies became available. With the arrival of strong, permanent magnets it became possible to assemble compact and self sustained spectrometers consisting of the central rod protecting the silicon detector from the direct radiation from the source and a ring of magnetic blades attached to the central rod to bend the trajectories of electrons towards the detector [8]. These mini-orange spectrometers were often operated in sets as each covered only a limited momentum range. The small size and a carefree operation were at the same time the strongest but also the only assets of mini-oranges.

The second novelty was the supercondicting magnet. The growing industrial and medical demand for such devices significantly reduced the price and made them available to the scientific community at large. As the result many groups world-wide opted for building their conversion electron spectrometers using such magnets. They offered uniform and strong magnetic fields (up to several Tesla) assuring very high transport efficiencies when used in the solenoid mode. The ability to profile the shape of the field by changing the current in the selected coils gave the addition degree of trajectory control. However all these benefits were to some degree compromised by the costs and labor to maintain the coils in the liquid helium temperature.

Finally, the enormous explosion of easily accessible computing power brought by the advent of PCs allowed for creative design of iron-free non-superconducting coils for magnetic spectrometers. After thorough consideration this option was chosen for ELLI.

\section{ELLI spectrometer}

ELLI stands for Electron Lens for IGISOL. ELLI was the first conversion electron spectrometer that was designed and build for the detection, identification, and study of exotic isotopes separated with IGISOL. To work with mass separated low-intensity radioactive beams the spectrometer had to have high efficiency and incorporate a tape drive to remove the buildup of background activities, see Fig. 1. It was desired to have a provision for up to three detectors allowing for simultaneous measurements of conversion electrons, betas, and gamma- or X-rays. Since oil-free vacuum was still a rarity at that time, the cooled detectors required a cold trap. To tune the separator with a stable ion beam, a provision for a removable Faraday cup had to be provided to place it just in front of the implantation point. Further, compatibility with the existing power sources and detector support systems was required. ELLI 
is described in details in Ref. [9]. As is the case for all solenoid spectrometers, the detection efficiency is a function of the current and electron energy ranging from $15 \%$ at $250 \mathrm{keV}$ to $5 \%$ at $2 \mathrm{MeV}$. The overall background reduction as compared with a plain silicon detector in close geometry is about a factor of 4 .

ELLI design included several new solutions; for instance, the abandonment of the axial implantation of the beam. As a rule, all previous designs of similar devices had the beam enter along the symmetry axis through the hole in the detector. Such annular detectors are very expensive and their performance (resolution and noise level) is often inferior to the regular detectors of the same size. Actually, thanks to the large mass difference between electrons and nuclei there is no need to worry too much about the deflection of the ions in the magnetic fields chosen for bending of electron trajectories. In case of ELLI separated beam enters at a steep 45 degree angle through the gap between the two coils arranged in the Helmholtz configuration. This novel idea, invented for ELLI, of the beam entering at an oblique angle with respect to the symmetry axis of the magnetic field has been subsequently adopted by SACRED [10] and SAGE [11] spectrometers.

\section{Measurements with ELLI}

The first accelerator at JYFL (the MC-20 cyclotron) has stopped operation in 1991 and the new $\mathrm{K}=130$ became operational in 1993. The tests, commissioning, and the first measurements with ELLI were made still in the old laboratory and continued with the beams from the new cyclotron.

Among the first reported results was the beta decay of ${ }^{114} \mathrm{Ru}$ and $Q_{\beta}$ systematics for neutron-rich $\mathrm{Ru}$ isotopes [12], the beta decay of ${ }^{113} \mathrm{Rh}$ and the observation of ${ }^{113 \mathrm{~m}} \mathrm{Pd}$ [13], Gamow-Teller decay of ${ }^{118} \mathrm{Pd}$ and the new isotope ${ }^{120} \mathrm{Pd}$ [14], deformation studies in the neutron-rich $\mathrm{Zr}$ region [15], and the decay of ${ }^{108} \mathrm{Mo}$ and of the neighboring even Mo-isotopes [16]. At the new cyclotron a new isomer in ${ }^{125} \mathrm{La}$ was discovered [17], isomeric state of ${ }^{80} \mathrm{Y}$ and its role in the astrophysical rp-process was investigated [18], as well as isomers of astrophysical interest in neutron-defficient nuclei at masses $A=81,85$ and 86 [19]. ELLI was even used at ISOLDE, CERN in the investigation of nonanalog beta decay of ${ }^{74} \mathrm{Rb}[20]$. The latest experiment using ELLI was an offline measurement of ${ }^{133 \mathrm{~m}}$ Xe conversion coefficient from a source produced and implanted at IGISOL [21]. This particular isomer is important in indication of nuclear explosion and is therefore monitored by Comprehensive Nuclear-TestBan Treaty Organization (CTBTO) with sampling stations located around the globe.

The installation of the cooler and the Penning trap at the start of the new millennium brought new, unique research possibilities to IGISOL at the same time contributing to the demise of the role of the traditional spectroscopic methods. Nevertheless, after two decades of service ELLI remains in the 


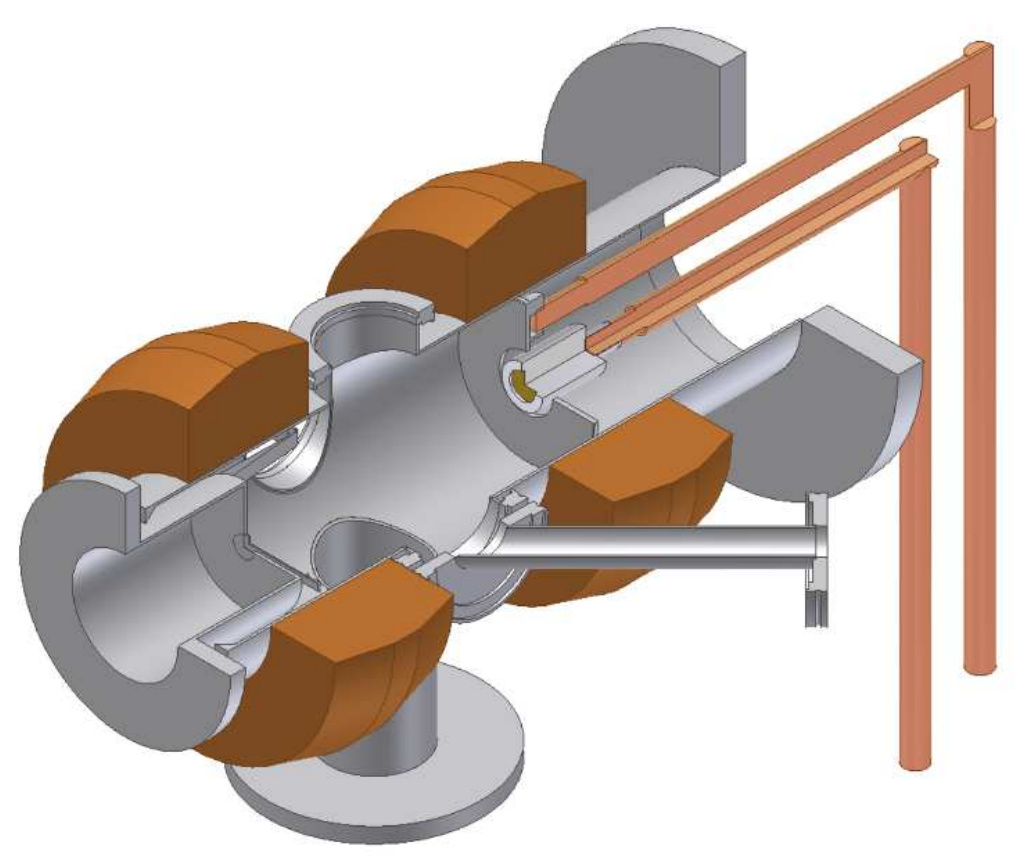

Fig. 1 ELLI spectrometer. The beam enters through the angled beam tube and is implanted in to a movable tape in the centre of the left hand side coil. The cooled $\mathrm{Si}(\mathrm{Li})$ detector is facing the implantation point in the centre of the other coil. Surrounding the $\mathrm{Si}(\mathrm{Li})$ detector is the cold trap to collect any oil vapour from the vacuum chamber before cooling the detector. Other detectors (Si for $\beta$ and Ge/LEGe for $\gamma$ and X-rays) can be inserted into the recessed aluminium cup giving access close to the implantation point. The removable faraday cup for beam tuning is not pictured here but it can be rotated in and out of the beam without breaking the vacuum.

working condition awaiting new challenges at JYFL or experiments at other facilities.

\section{In-trap conversion electron spectroscopy}

As mentioned above with the arrival of RFQ cooler and JYFLTRAP Penning trap the main focus of the IGISOL physics program turned towards utilization of ion traps in beam preparation and performing atomic mass measurements. Right from the trap design phase the idea was to harness the high mass resolving power of a Penning trap to benefit the decay spectroscopy both after the trap as well as inside the trap [22].

The concept of an ideal trapped decay source was first developed for precision study needed for search of scalar and tensor currents in weak interaction. In practise the trapped source technique allows for detection of the beta-decay daughter ion recoiling out of the trap. Examples of this kind of ion trap in- 
stallations are LPC trap at Caen [23] and WITCH trap at ISOLDE [24], the former is a transparent Paul trap and the latter is an open Penning trap. The benefit of a trapped decay source was also recognised for high precision decay spectroscopy. As the trapped ions confined by magnetic and electric fields are emitting conversion electrons the scattering and energy loss in the backing material present in an implanted source are avoided. Furthermore, the strong magnetic field of a Penning trap can be employed to transport electrons efficiently into a small area high resolution detector and thus suppressing $\gamma$ - or X-ray background.

As described in the previous chapters the conversion electron detection efficiency over $\gamma$ - or X-ray efficiency is usually increased by introduction of guiding magnetic solenoid field. The effect of the magnetic field is two fold in the case of an implanted source. It increases the electron collection efficiency by capturing and guiding electrons emitted within a certain cone to the detector. On the other hand some of the electrons emitted to larger angles lose considerable portion of their energy in the backing material which shows up in the energy spectrum as a low energy tail of electron peaks. The deterioration of the spectrum becomes more evident with increasing magnetic field and source implantation depth. With a trapped electron source there is not such limitation.

The first feasibility test of in-trap conversion electron spectroscopy was carried out at ISOLDE, CERN using REX-ISOLDE accumulator Penning trap REXTRAP to confine the source ions. A EB10GC-500P detector assembly with a low noise PA1201 preamplifier was acquired from Canberra Semiconductor for the test. The $500 \mu \mathrm{m}$ thick detector has $10 \mathrm{~mm}^{2}$ active area $(3.3$ mm diameter) and dead layer of $250 \AA$. The feasibility of the method was demonstrated by measuring isomeric decays of on-line separated ${ }^{116 \mathrm{~m}} \mathrm{In}$ and ${ }^{118 \mathrm{~m}}$ In sources [25]. The promising results encouraged to bring the detector setup to Jyväskylä where the foreseen physics program and expertise was more suited for further investigation of the method.

So far only one on-line test has been run at JYFLTRAP [26]. In total ten conversion electron emitting isomers of neutron-rich isotopes produced in proton induced fission of ${ }^{238} \mathrm{U}$ were studied. Among these was the same $118 \mathrm{~m}_{2} \mathrm{In}$ isomer studied earlier at ISOLDE. Compared to the first test some improvement could be seen in the resolution. The showcase of the Jyväskylä test experiment was the detection of conversion electrons at $9.9 \mathrm{keV}$ from short lived ${ }^{117 \mathrm{~m}} \mathrm{Pd}$ isomeric state with half-life of only $19.1 \mathrm{~ms}$. Electron energy spectrum from another measured isomeric decay of ${ }^{118 \mathrm{~m}} \mathrm{Ag}$ is shown in Fig. 2. The largest peaks are K, L, and M conversion electron lines of the $127.0 \mathrm{keV}$ transition at $102.2 \mathrm{keV}, 123.9 \mathrm{keV}$, and $127.0 \mathrm{keV}$, respectively. The measured peaks exhibit an outstanding energy resolution, taking a $2.2 \mathrm{keV}$ resolution for the $102.2-\mathrm{keV}$ peak as an example. The small peaks at slightly higher energies arise from isomeric decay of ${ }^{118 \mathrm{~m}_{2}} \mathrm{In}$ that was present in the trap as contamination. At the low end of the spectrum Auger electron peaks are visible. From the spectrum one can see that besides the very good energy 


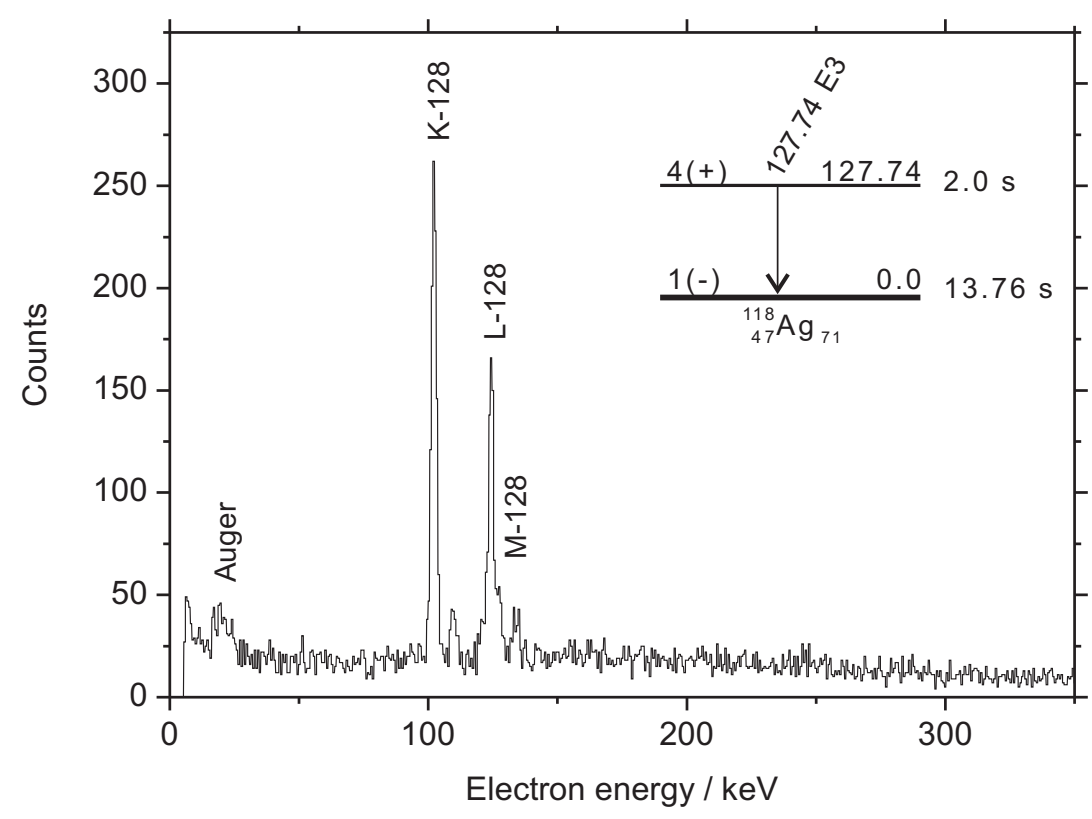

Fig. 2 In-trap electron spectrum recorded for ${ }^{118 \mathrm{~m}} \mathrm{Ag}$. The K, L, and M conversion electron lines of the $127.7 \mathrm{keV}$ transition are labelled. The inset shows the studied isomeric transition in ${ }^{118} \mathrm{Ag}$.

resolution the overall background level and the energy threshold are quite low.

Although the first test experiments have been succesfull there are still some problems related to this technique. The confinement of electrons with high magnetic field all the way to the detector enhances the backscattering which is roughly proportional to the square of the electron impact angle.

Compared to the ELLI spectrometer the in trap electron spectroscopy method is still lacking simultaneous gamma-ray detection. Some issues with detector contamination by recoiling highly charged radioactive ions was suspected in the REXTRAP experiment. Furthermore the tuning of the Penning trap becomes challenging when the extraction side is blocked with electron detector. Due to these complications, of which some could be overcome by investing efforts to $R \& D$, the studied physics case should be such that it is impossible or hard to be tackled with traditional techniques. The experimental target could be ultra-high-resolution electron spectroscopy or detection of very low-energy electrons. The problem could also be turned around in a way that conversion electron detection would be used to image the trapped ion cloud and hence to diagnose the trapping process itself. 


\section{Future of conversion electron spectroscopy at IGISOL}

As it has been seen in the decay-spectroscopy experiments concentrating in gamma or neutron detection, the trend has been towards utilizion of the Penning trap as a high-resolution mass filter to provide even isomerically pure sources for experiments. The same is foreseen also for the conversion electron spectroscopy. One option for this kind of experiment would be to install the ELLI spectrometer behind the trap but also other detectors without a magnetic lens could be considered.

For the in-trap technique to be used in more demanding decay studies it probably requires some further development as was outlined in the Ref. [26]. A more sophisticated in-trap electron detector with a larger area and segmentation would allow coincidence detection between electrons. An easy insertion and retraction of the detector without breaking the vacuum would be desirable not least for the trap tuning point of view but it also would allow quick checking of emitted conversion electrons in the midst of a post-trap spectroscopy experiment. The data readout and acquisition from the electron detector could be simplified by operating the trap in the ground potential rather than the presently used high-voltage platform. This can be achieved by use of a pulsedown drift-tube in the trap injection line. The ability to detect coincident electromagnetic radiation together with the conversion electrons would enhance the applicability of the technique. A limited choise of gamma-ray detectors can be considered due to the tight space limitation and high magnetic field inside the trap magnet. An option with a reasonable resolution would be an array of $\mathrm{LaBr}$ crystals combined with silicon based photomultipliers surrounding the trapping region in a close geometry.

Conversion electron spectroscopy has had an important role throughout the life of decay spectroscopy at IGISOL. It all started with a simple silicon detector and tape transport system, followed by a specialiced ELLI electron spectrometer and has now evolved into a more complex in-trap and post-trap spectroscopy set-ups. Surely in the future the conversion electron spectroscopy will have its place in the decay spectroscopic studies carried out at the IGISOL facility independent of the techniques used for preparing the source or detecting the electrons.

Acknowledgements This work has been supported by the Academy of Finland under the Finnish Centre of Excellence Programme 2000-2005 (Project No. 44875, Nuclear and Condensed Matter Physics Programme at JYFL)

\section{References}

1. P. Kleinheinz, L. Samuelsson, R. Vukanovic, K. Siegbahn, Nucl. Instrum. Methods Phys. Res. 32, 1 (1965)

2. L. Westerberg, L. Edvardson, G. Madueme, J. Thun, Nucl. Instrum. Methods Phys. Res. 128, 61 (1975)

3. J. Kantele, M. Luontama, A. Passoja, R. Julin, Nucl. Instrum. Methods Phys. Res. 130, $467(1975)$ 
4. R. Julin, J. Kantele, J. Kumpulainen, M. Luontama, A. Passoja, W. Trzaska, E. Verho, Phys. Rev. C 36, 1129 (1987)

5. R. Julin, J. Kantele, J. Kumpulainen, M. Luontama, V. Nieminen, A. Passoja, W. Trzaska, E. Verho, J. Blomqvist, Nucl. Instrum. Methods Phys. Res. A 270, 74 (1988)

6. J. Ärje, J. Äystö, H. Hyvönen, P. Taskinen, V. Koponen, J. Honkanen, K. Valli, A. Hautojärvi, K. Vierinen, Nucl. Instrum. Methods Phys. Res. A 24, 774 (1986)

7. J. Äystö, C. Davids, J. Hattula, J. Honkanen, K. Honkanen, P. Jauho, R. Julin, S. Juutinen, J. Kumpulainen, T. Lönnroth, A. Pakkanen, A. Passoja, H. Penttilä, P. Taskinen, E. Verho, A. Virtanen, M. Yoshii, Nucl. Phys. A 480, 104 (1988)

8. J. van Klinken, S. Feenstra, G. Dumont, Nucl. Instrum. Methods 151, 433 (1978)

9. J. Parmonen, Z. Janas, W. Trzaska, J. Äystö, J. Kantele, P. Jauho, A. Jokinen, H. Penttilä, Nucl. Instrum. Methods Phys. Res. A 306, 504 (1991)

10. H. Kankaanpää, et al., Nucl. Instrum. Methods Phys. Res. A 534, 503 (2004)

11. P. Papadakis, et al., Journal of Physics: Conference Series 312, 052017 (2011)

12. A. Jokinen, J. Äystö, P.P. Jauho, M. Leino, J.M. Parmonen, H. Penttilä, K. Eskola, Z. Janas, Nucl. Phys. A 549, 420 (1992)

13. H. Penttilä, T. Enqvist, P. Jauho, A. Jokinen, M. Leino, J. Parmonen, J. Äystö, K. Eskola, Nucl. Phys. A 561, 416 (1993)

14. Z. Janas, J. Äystö, K. Eskola, P.P. Jauho, A. Jokinen, J. Kownacki, M. Leino, J.M. Parmonen, H. Penttilä, J. Szerypo, J. zylicz, Nucl. Phys. A 552, 340 (1993)

15. G. Lhersonneau, B. Pfeiffer, K.L. Kratz, T. Enqvist, P.P. Jauho, A. Jokinen, J. Kantele, M. Leino, J.M. Parmonen, H. Penttilä, J. Äystö, Phys. Rev. C 49, 1379 (1994)

16. A. Jokinen, T. Enqvist, P.P. Jauho, M. Leino, J.M. Parmonen, H. Penttilä, J. Äystö, K. Eskola, Nucl. Phys. A 584, 489 (1995)

17. G. Canchel, R. Béraud, E. Chabanat, A. Emsallem, N. Redon, P. Dendooven, J. Huikari, A. Jokinen, V. Kolhinen, G. Lhersonneau, M. Oinonen, A. Nieminen, H. Penttilä, K. Peräjärvi, J. Wang, J. Äystö, Eur. Phys. J. A 5, 1 (1999)

18. Y. Novikov, H. Schatz, P. Dendooven, R. Braud, C. Mieh, A. Popov, D. Seliverstov, G. Vorobjev, P. Baumann, M. Borge, G. Canchel, P. Desagne, A. Emsallem, W. Huang, J. Huikari, A. Jokinen, A. Knipper, V. Kolhinen, A. Nieminen, M. Oinonen, H. Penttilä, K. Peräjärvi, I. Piqueras, S. Rinta-Antila, J. Szerypo, Y. Wang, J. Äystö, Eur. Phys. J. A 11, 257 (2001)

19. A. Kankainen, G. Vorobjev, S. Eliseev, W. Huang, J. Huikari, A. Jokinen, A. Nieminen, Y. Novikov, H. Penttilä, A. Popov, S. Rinta-Antila, H. Schatz, D. Seliverstov, Y. Suslov, J. Äystö, Eur. Phys. J. A 25, 355 (2005)

20. M. Oinonen, J. Äystö, P. Baumann, J. Cederkäll, S. Courtin, P. Dessagne, S. Franchoo, H. Fynbo, M. Górska, J. Huikari, A. Jokinen, A. Knipper, U. Köster, G.L. Scornet, C. Miehé, A. Nieminen, T. Nilsson, Y. Novikov, K. Peräjärvi, E. Poirier, A. Popov, D. Seliverstov, T. Siiskonen, H. Simon, O. Tengblad, P.V. Duppen, G. Walter, L. Weissman, K. Wilhelmsen-Rolander, Phys. Lett. B 511, 145 (2001)

21. K. Peräjärvi, J. Turunen, J. Hakala, A. Jokinen, I.D. Moore, H. Penttilä, A. Saastamoinen, T. Siiskonen, H. Toivonen, J. Äystö, App. Rad. Isotopes 66, 530 (2008)

22. J. Szerypo, A. Jokinen, V. Kolhinen, A. Nieminen, S. Rinta-Antila, J. Äystö, Nucl. Phys. A 701, 588 (2002). 5th International Conference on Radioactive Nuclear Beams

23. D. Rodríguez, A. Méry, G. Ban, J. Brégeault, G. Darius, D. Durand, X. Fléchard, M. Herbane, M. Labalme, E. Linard, F. Mauger, Y. Merrer, O. Naviliat-Cuncic, J. Thomas, C. Vandamme, Nucl. Instrum. Methods Phys. Res. A 565, 876 (2006)

24. M. Beck, F. Ames, D. Beck, G. Bollen, B. Delauré, V.V. Golovko, V.Y. Kozlov, I.S. Kraev, A. Lindroth, T. Phalet, W. Quint, P. Schuurmans, N. Severijns, B. Vereecke, S. Versyck, Nucl. Instrum. Methods Phys. Res. A 503, 567 (2003)

25. L. Weissman, F. Ames, J. Äystö, O. Forstner, K. Reisinger, S. Rinta-Antila, Nucl. Instrum. Methods Phys. Res. A 492, 451 (2002)

26. J. Rissanen, V. Elomaa, T. Eronen, J. Hakala, A. Jokinen, S. Rahaman, S. Rinta-Antila, J. Äystö, Eur. Phys. J. A 34, 113 (2007) 